\title{
Actualización sobre el papel de la terapia nutricional en pacientes sometidos a trasplante renal: de la enfermedad renal al trasplante
}

\author{
Fernanda I. Ramírez-Casale ${ }^{1}$, Enrique Cervantes-Pérez ${ }^{1,2,3,4 *}$, Miguel Robledo-Valdez ${ }^{1}$, \\ Ma. Fernanda Padilla-Rubio ${ }^{1}$, Astrid Villaseñor-Ochoa ${ }^{1}$, Gabino Cervantes-Guevara ${ }^{2,3,5}$, \\ Guillermo A. Cervantes-Cardona ${ }^{6}$, Sol Ramírez-Ochoa ${ }^{4}$, Adriana Nápoles-Echauri ${ }^{6}$, \\ Gabino Cervantes-Pérez ${ }^{5}$ y Josué I. Ruiz-Gallardo ${ }^{7}$
}

${ }^{1}$ Departamento de Nutriología Clínica, Instituto Nacional de Ciencias Médicas y Nutrición Salvador Zubirán, Ciudad de México; ${ }^{2}$ Departamento de Bienestar y Desarrollo Sustentable, Centro Universitario del Norte, Universidad de Guadalajara, Colotlán, Jalisco; ${ }^{3}$ Departamento de Clínicas Médicas, Centro Universitario de Ciencias de la Salud, Universidad de Guadalajara, Guadalajara, Jalisco; ${ }^{4}$ Departamento de Medicina Interna, Hospital Civil de Guadalajara Fray Antonio Alcalde, Guadalajara, Jalisco; ${ }^{5}$ Departamento de Gastroenterología, Hospital Civil de Guadalajara Fray Antonio Alcalde, Guadalajara, Jalisco; ${ }^{6}$ Departamento de Disciplinas Filosóficas, Metodológicas e Instrumentales, Centro Universitario de Ciencias de la Salud, Universidad de Guadalajara, Guadalajara, Jalisco; ${ }^{7}$ Departamento de Nefrología, Hospital Regional Valentín Gómez Farias, Instituto de Seguridad y Servicios Sociales de los Trabajadores del Estado, Guadalajara, Jalisco. México

\section{Resumen}

La enfermedad renal crónica (ERC) tiene implicaciones directas sobre el estado nutricional, provocando anorexia y catabolismo muscular, siendo más frecuente en los pacientes con terapia de remplazo renal. La importancia de la evaluación nutricional en pacientes con ERC sometidos a trasplante renal (TR) es cada vez mayor, sin embargo, la evidencia en relación con la herramienta de evaluación nutricional que utilizar es escasa. Las guías actuales sugieren el uso del puntaje de inflamación de desnutrición (MIS) para identificar a los pacientes con mayor riesgo nutricional, disminuyendo así el riesgo de complicaciones quirúrgicas en los pacientes sometidos a TR. Así mismo, la suplementación con ciertos micronutrientes son parte fundamental en la prevención y tratamiento de alteraciones metabólicas secundarias al trasplante. La implementación de la terapia médico-nutricional en pacientes pretrasplantados y postrasplantados forma parte fundamental en el manejo multidisciplinario, siendo la mejor estrategia preventiva para la preservación de la función renal, en conjunto con la terapia farmacológica y ejercicio físico, beneficiando así al huésped y al injerto.

Palabras clave: Trasplante renal. Enfermedad renal. Desnutrición. Soporte nutricional.

\section{An update on the role of nutritional therapy in patients undergoing kidney transplantation: from kidney disease to transplantation}

\section{Abstract}

Chronic kidney disease (CKD) has direct implications on nutritional status, resulting in anorexia and muscle catabolism, being more frequent in patients with renal replacement therapy. The importance of nutritional assessment in CKD patients undergoing kidney transplantation (KT) is increasing, however, the evidence regarding the nutritional assessment tool is

\section{Correspondencia:}

*Enrique Cervantes-Pérez

E-mail: enrique.cervantes@academico.udg.mx licencia CC BY-NC-ND (http://creativecommons.org/licenses/by-nc-nd/4.0/).
Fecha de recepción: 25-08-2020

Fecha de aceptación: 12-10-2021

DOI: 10.24875 /NEFRO.20000045
Disponible en internet: 27-12-2021

Nefro Latinoam. 2021;18:148-155 www.nefrologialatinoamericana.com 
lacking. Current guidelines suggest the use of the Malnutrition Inflammation Score (MIS) to identify patients with nutritional risk, thus reducing the risk of surgical complications in patients undergoing KT. Likewise, supplementation with certain micronutrients is a fundamental part in the prevention and treatment of metabolic disorders associated to transplantation. The implementation of medical nutrition therapy in pre and post-transplant patients is an essential part of the multidisciplinary management, being the best preventive strategy for the preservation of kidney function, along with drug therapy and physical exercise, benefiting the host and the graft.

Key words: Kidney transplantation. Kidney disease. Malnutrition. Nutritional support.

\section{Introducción}

El trasplante renal (TR) es una opción de tratamiento exitoso para pacientes con enfermedad renal en etapa terminal, ya que mejora la supervivencia y la calidad de vida de los pacientes en comparación con la terapia de reemplazo renal ${ }^{1}$. Aun cuando el grupo de trabajo Kidney Disease Improving Global Outcomes (KDIGO) desarrolla pautas basadas en la prevención de complicaciones posteriores al TR, no describe de manera específica recomendaciones nutricionales, las cuales deben tomarse en cuenta en todo paciente que se someterá a TR.

Debido a la liberación de las restricciones dietéticas y los inevitables efectos secundarios metabólicos de los fármacos inmunosupresores, el abordaje nutricional es de gran relevancia, ya que es indispensable para la prevención y/o retraso de la progresión de la enfermedad, tanto de la nefropatía crónica del injerto como de varios otros eventos adversos posteriores al trasplante, siendo las más frecuentes: enfermedad cardiovascular (ECV), dislipidemia, diabetes de nueva aparición posterior al trasplante e incluso la pérdida de tejido óseo ${ }^{2}$. Es por ello que el manejo nutricional sigue siendo la mejor estrategia preventiva rentable, junto con la adherencia farmacológica y ejercicio, beneficiando al injerto y preservando la función renal a largo plazo ${ }^{3,4}$.

Aun cuando existen estudios que describen el aumento de peso posterior al TR, existen pautas limitadas en relación con la dieta o la actividad física para mitigar la ganancia de peso no deseada ${ }^{2}$. No obstante, no solo existe la predisposición al aumento de peso, sino también la posible presencia de desnutrición, la cual se asocia con la progresión a diálisis peritoneal empeorando el estado nutricional, junto con inflamación crónica y arteriosclerosis ${ }^{1}$.

Es imperativo establecer objetivos nutricionales previos y posteriores al trasplante con el fin de corregir la desnutrición, sobrepeso u obesidad, mejorando la sobrevida del paciente, así como la adaptación del injerto renal y promoviendo mejorarías en las concentraciones séricas de albúmina, glucosa, creatinina y hemoglobina, atenuando el catabolismo proteico ${ }^{5}$.

\section{Evaluación nutricional}

Independientemente de la etapa del TR, es recomendable que la valoración nutricional se realice de manera periódica con intervalos no mayores a seis meses; los parámetros que evaluar durante esta deben basarse en entrevistas tanto al paciente como a familiares, registros alimentarios, así como anamnesis médica, social y nutricional. El examen físico, la evaluación de parámetros de laboratorio, la interpretación de los cambios en el apetito y la medición de variables antropométricas también forman parte de esta valoración ${ }^{6}$.

Utilizando componentes de la valoración global subjetiva (VGS), Kalantar et al. desarrollaron un sistema de puntuación cuantitativa de desnutrición para pacientes en diálisis (Dialysis Malnutrition Score [DMS]), el cual consta de siete aspectos: cambio de peso, ingesta de alimentos, síntomas gastrointestinales, capacidad funcional, comorbilidades, grasa subcutánea y signos de desgaste muscular; no obstante, se eliminaron la ascitis y el edema, y se agregó el número de años de terapia de diálisis al componente de comorbilidad ${ }^{7}$. Sin embargo, el DMS no se correlacionó con ningún parámetro objetivo de la evaluación nutricional para pacientes en espera de TR, a excepción de la capacidad total de fijación de hierro, donde se observó una correlación entre la duración de la hemodiálisis y el sexo; cabe señalar que el DMS resulta más objetivo que la VGS convencional con pautas fáciles y prácticas ${ }^{8}$.

En los últimos años, las estimaciones de prevalencia derivadas de la VGS y el puntaje de inflamación de desnutrición (MIS) ${ }^{9}$ han sido utilizadas en la literatura, debido a que la VGS es un puntaje de evaluación nutricional validado y establecido ampliamente, utilizado internacionalmente en diversas disciplinas además de la nefrología; por otra parte, el MIS es un puntaje semicuantificado basado en la VGS, específico para 
pacientes con enfermedad renal crónica $(E R C)^{10}$. Recientemente, las guías Kidney Disease Outcomes Quality Initiative (KDOQI) 2020 mencionan la utilidad del MIS como predictor significativo de mortalidad a 2.97 años ( $p<0.001)$; a su vez, es catalogado como la mejor herramienta predictiva de mortalidad por todas las causas (p. ej., eventos cardiovasculares) en pacientes que reciben terapia con hemodiálisis ${ }^{11}$. No obstante, las mismas guías concluyen que existe evidencia limitada para sugerir el uso de un sola herramienta de tamizaje para identificar adultos con ERC o postrasplantados en riesgo de desnutrición ${ }^{11}$.

Otras herramientas actuales disponibles en la evaluación nutricional es la determinación de la composición corporal total por medio de la bioimpedancia. En un estudio realizado por Sukackiene et al. se observó que un mayor índice de edema (calculado como la relación entre el agua extracelular [ECW] y el agua corporal total [TBW]) se relacionó con mayor desnutrición de acuerdo con la VGS ${ }^{12}$; esto es de gran utilidad, ya que este fenómeno se ha presentado también en la enfermedad renal poliquística autosómica dominante donde los valores más altos de ECW/TBW se correlacionaron con una puntuación menor de VGS ${ }^{13}$.

En pacientes con TR, el índice de masa corporal (IMC) ha demostrado ser un predictor de supervivencia del injerto, incrementando el riesgo de rechazo con valores bajos o elevados de IMC ${ }^{5}$. Así mismo, la medición de albúmina sérica en correlación con el valor de proteína $C$ reactiva (PCR) es directamente proporcional a la ingesta proteica y se incluye por los diferentes consensos como parte del diagnóstico del déficit proteico ${ }^{6}$. Aunque, en la práctica clínica, las pruebas útiles en la valoración de la función renal son la creatinina sérica, el nitrógeno ureico y la tasa de la filtración glomerular, es de suma importancia considerar la masa muscular, la ingesta proteica y el estado funcional del riñón a la hora de hablar del estado nutricional del paciente ${ }^{14}$. Por lo tanto, el estado nutricional en los candidatos a TR debe evaluarse de manera más precisa debido a las consecuencias específicas tanto en el periodo previo como posterior al trasplante.

\section{Pretrasplante}

La pérdida de peso previa al trasplante se asocia con un riesgo relativamente alto de mortalidad después del trasplante. En pacientes con diálisis, sin un programa específico de actividad física, la pérdida de peso está asociada con la pérdida de masa corporal magra, relacionada con un mayor riesgo de mortalidad. Por el contrario, una mayor cantidad de masa muscular previa al trasplante, reflejada por niveles altos de creatinina sérica, se asocia con una mayor supervivencia después del TR ${ }^{15}$.

Dados los datos sobre el efecto de la obesidad previa al trasplante, un estudio reciente exploró el efecto de la composición corporal en las tasas de supervivencia del injerto a largo plazo, demostrando que la obesidad sarcopénica pretrasplante y postrasplante se asocia con una mayor mortalidad y falla del injerto, definiendo a la obesidad sarcopénica como masa muscular reducida pretrasplante representada por una creatinina sérica más baja en pacientes con un $I M C \geq 30 \mathrm{~kg} / \mathrm{m}^{2} 16,17$. Aunque se recomienda que los pacientes obesos pierdan peso, esto debe ser transitorio, debido al riesgo de pérdida de masa magra y aumento de masa grasa que presenta este grupo en especial ${ }^{17}$. A su vez, aqueIlos pacientes con IMC $<23 \mathrm{~kg} / \mathrm{m}^{2}$ se asociaron a una menor supervivencia a largo plazo, relacionado principalmente a una baja masa corporal magra y por consecuencia a pobres resultados ${ }^{15}$.

Es por eso que durante este periodo el receptor del injerto necesita aumentar adecuadamente la ingesta energética de $35-40 \mathrm{kcal} / \mathrm{kg} / \mathrm{día}$ y de proteína hasta $1.4 \mathrm{~g} / \mathrm{kg} / \mathrm{día}$, durante al menos 4 semanas, no solo para igualar el catabolismo proteico seguido a la administración de esteroides y al estrés quirúrgico, sino también para prevenir o tratar la desnutrición, lo que llevaría a una cicatrización deficiente de heridas, así como a mayor susceptibilidad a infecciones ${ }^{3}$.

\section{Postrasplante}

Después del TR, la evaluación nutricional es crucial para retrasar el deterioro de la función renal de los pacientes y reducir los trastornos metabólicos asociados. Posterior al trasplante, y bajo en escenario sin complicaciones, la ingesta oral debe reanudarse dentro de las primeras 24 a 48 horas y se puede reestablecer una dieta regular dentro de la primera semana. En términos generales, con un injerto funcional y sin complicaciones tempranas asociadas, las restricciones dietéticas experimentadas durante la terapia de diálisis se pueden abandonar rápidamente y se puede proponer adoptar un nuevo plan de alimentación saludable. Aunque es necesario tomar en cuenta que el apetito y el gusto de los pacientes en general mejoran rápidamente y pueden verse potenciados por el estrés metabólico quirúrgico y las altas dosis de corticosteroides. Estos dos últimos factores pueden aumentar el gasto energético temprano postrasplante $y$, por lo tanto, 
determinar una mayor demanda nutricional ${ }^{18}$. Además, las dosis altas de corticosteroides aceleran la tasa catabólica de proteínas y frecuentemente crean o empeoran el balance nitrogenado negativo ${ }^{19}$.

Por otro lado, dicha mejoría del apetito puede propiciar un aumento de peso, lo que lleva a una gran cantidad de desafíos nutricionales que requieren un control dietético estrecho. Así mismo, se sabe que los inmunosupresores empleados inducen efectos adversos metabólicos, como hipercatabolismo proteico, hiperlipidemia, intolerancia a la glucosa, hiperpotasemia, hipofosfatemia, hipomagnesemia y obesidad ${ }^{20}$.

En el periodo inicial posterior al trasplante, el desafío nutricional es contrarrestar los efectos metabólicos antes mencionados ${ }^{20}$. Algunos de los objetivos principales son:

- Mantenimiento de las reservas de proteínas viscerales a pesar del catabolismo proteico.

- Promoción de la cicatrización de heridas.

- Prevención de infecciones asociadas a la cirugía y los inmunosupresores.

- Prevención de complicaciones electrolíticas que acompañan cambios rápidos en la función renal.

Las recomendaciones de proteínas inmediatamente después del procedimiento quirúrgico oscilan entre 1.2 y $2 \mathrm{~g} / \mathrm{kg} / \mathrm{día}$, ya que se ha demostrado que una ingesta proteica por debajo de $1 \mathrm{~g} / \mathrm{kg} /$ día en el postoperatorio puede conducir a un balance negativo de nitrógeno y aumentar la pérdida de masa muscular ${ }^{17,18}$.

Más tarde, se debe optimizar el estado nutricional, incluido el aumento de peso, el control de la obesidad y el control lipídico ${ }^{20}$. Para lograr esto, el manejo nutricional tardío (4-6 semanas) al trasplante tiene como objetivos:

- Lograr y mantener un buen estado nutricional en general.

- Erradicar o disminuir la obesidad, dislipidemia e hipertensión.

- Promover el control glucémico y el manejo de diabetes mellitus.

- Prevención y manejo de la enfermedad mineral ósea.

- Prevención y gestión de deficiencias nutricionales.

A pesar de las recomendaciones altas en proteína en una etapa temprana para revertir el balance de nitrógeno y aumentar la masa muscular, no hay evidencia clara sobre el requerimiento de proteínas en receptores estables, siendo el objetivo optimizar el estado nutricional y evitar una carga proteica innecesaria al riñón, la cual puede definirse en $0.75 \mathrm{~g} / \mathrm{kg} / \mathrm{día}$ en mujeres y $0.84 \mathrm{~g} / \mathrm{kg} / \mathrm{día}$ en hombres en la etapa tardía, independientemente de la función renal ${ }^{3,17}$ (Tabla 1).
Tabla 1. Recomendaciones nutricionales en adultos receptores de trasplante renal

\begin{tabular}{|c|c|}
\hline $\begin{array}{l}\text { Proteína } \\
\text { Etapa temprana (inmediatamente } \\
\text { después de la cirugía) } \\
\text { Etapa tardía } \\
\text { (4-6 semanas) }\end{array}$ & $\begin{array}{c}1.2-2.0 \mathrm{~g} / \mathrm{kg} \text { peso } \\
\text { corporal/día } \\
0.8-1.0 \mathrm{~g} / \mathrm{kg} \text { peso } \\
\text { corporal/día }\end{array}$ \\
\hline $\begin{array}{l}\text { Calorías } \\
\text { Etapa temprana } \\
\text { Etapa tardía }\end{array}$ & $\begin{array}{l}\text { 30-35 kcal/día } \\
20-35 \mathrm{kcal} / \mathrm{día}\end{array}$ \\
\hline Grasas & $30-35 \%$ de VET \\
\hline Carbohidratos & $50-60 \%$ de VET \\
\hline Fibra & 25-30 g/día \\
\hline Potasio & 2-4 g/día \\
\hline Sodio & 2-4 g/día \\
\hline Fósforo & $\begin{array}{l}\text { Individualizar con base } \\
\text { en los niveles séricos }\end{array}$ \\
\hline $\begin{array}{l}\text { Líquidos } \\
\text { Etapa temprana } \\
\text { Etapa tardía }\end{array}$ & $\begin{array}{l}\text { Limitar en presencia de } \\
\text { edema o sobrecarga de } \\
\text { volumen. } \\
\text { Irrestrictos a menos que } \\
\text { haya sobrecarga }\end{array}$ \\
\hline Alcohol & 2 bebidas estándar/día \\
\hline
\end{tabular}

VET: valor energético total.

Adaptada de Dionigi, et al., $2017^{19}$.

\section{Soporte nutricional}

Si la ingesta vía oral es insuficiente, puede ser necesario la utilización de suplementos nutricionales orales (SNO) para proporcionar el aporte suficiente de energía y proteínas. Si los pacientes persisten con ingesta alimentaria insuficiente y por consecuencia déficit calórico, el uso de nutrición enteral (NE) o parenteral (NP) está indicado.

El apoyo nutricional es una parte integral del manejo de pacientes críticos. Sin embargo, pocos estudios han demostrado la importancia de la intervención nutricional en receptores de $\operatorname{TR}^{21,22}$. Además, el soporte postoperatorio temprano mejora la cicatrización y disminuye las tasas de infección viral, sepsis y translocación bacteriana. Se sugiere que el uso de la NE se considere únicamente en casos seleccionados, si la ingesta es subóptima a pesar del uso de SNO. Es importante tener en cuenta las comorbilidades, el estado en general y las posibles perspectivas de supervivencia antes de iniciar la alimentación por dispositivos enterales (sondas de alimentación). Dichos escenarios incluyen pacientes que no logran consumir los nutrimentos 
adecuados por vía oral o experimentan complicaciones postoperatorias $^{23}$. Si bien no existen recomendaciones específicas en este tipo de pacientes, se recomienda el uso de fórmulas con alto contenido de nitrógeno para ayudar a promover la cicatrización de heridas y el anabolismo, prevenir infecciones, así como satisfacer las necesidades proteicas del paciente. La modificación de la fórmula será necesaria para los pacientes que experimenten una función tardía del injerto ${ }^{23}$. Sin embargo, cuando el tracto gastrointestinal no es funcional, o bien la NE no es suficiente para alcanzar los objetivos nutricionales, es pertinente el inicio de NP, ya sea total o parcial ${ }^{24}$, para lo cual se requiere una solución individualizada/personalizada, alta en proteínas, y de esta manera promover la cicatrización de heridas y el anabolismo. Los ajustes a los nutrimentos y al volumen en particular serán impulsados con base en la función del injerto, la producción de orina y si es necesaria o no la terapia de sustitución renal ${ }^{23}$.

\section{Alteraciones metabólicas}

EI TR corrige las anomalías asociadas y los efectos secundarios al tratamiento previo a la diálisis. Sin embargo, el trasplante por sí mismo, así como los efectos secundarios de los medicamentos inmunosupresores introducen un nuevo conjunto de anomalías metabólicas que pueden afectar significativamente los resultados de salud a corto y largo plazo de los pacientes y la supervivencia del injerto ${ }^{25}$. No obstante, los patrones dietéticos cambian con el tiempo y el monitoreo de los factores de riesgo para desarrollar complicaciones metabólicas después del trasplante debe ser una prioridad clínica e individualizada ${ }^{2,26}$.

El aumento de peso postrasplante, particularmente de grasa visceral, aumenta el riesgo de desarrollar diabetes de novo postrasplante, dislipidemia y ECV?2. Las dislipidemias se observan comúnmente después del TR y están relacionadas con la terapia inmunosupresora con corticoesteroides, ciclosporina y los inhibidores de la molécula diana de rapamicina. A su vez, se ha demostrado que la dislipidemia persiste más allá de los primeros meses postrasplante y, con ello, la leptina actúa sobre las células beta pancreáticas reduciendo la secreción de insulina, ocasionando resistencia a la insulina (RI), la cual tiene consecuencias perjudiciales asociadas a la disfunción del injerto y la $\mathrm{ECV}^{27}$.

Los factores de riesgo para desarrollar $\mathrm{RI}$ incluyen el uso de agentes inmunosupresores, obesidad y distribución central de grasa corporal, correlacionados
Tabla 2. Ingesta nutricional recomendada en pacientes trasplantados renales con dislipidemia

\begin{tabular}{|l|l|}
\hline Carbohidratos & $\begin{array}{l}\text { Consumirse preferentemente en forma de } \\
\text { granos enteros y de baja densidad } \\
\text { energética y/o índice glucémico }\end{array}$ \\
\hline $\begin{array}{l}\text { Fibra } \\
\text { Mujeres } \\
\text { Hombres }\end{array}$ & $25 \mathrm{~g}$ \\
\hline $\begin{array}{l}\text { Grasas } \\
\text { Saturadas } \\
\text { Monoinsaturadas } \\
\text { Polinsaturadas }\end{array}$ & $\begin{array}{l}\text { Hasta el 20\% } \\
\text { H-10\% }\end{array}$ \\
\hline Alcohol & $\begin{array}{l}\text { Limitar a no más de dos bebidas estándar } \\
\text { al día }\end{array}$ \\
\hline
\end{tabular}

VET: valor energético total.

Adaptada de Chadban, et al., $2010^{28}$

con los niveles de leptina en suero. Además, tanto la leptina como la RI están relacionadas con marcadores inflamatorios, como la PCR, la cual se ha sugerido como un marcador independiente de nefropatía crónica por injerto ${ }^{27}$.

Por lo tanto, es fundamental tomar en cuenta el estado nutricional posterior al trasplante, ya que también está determinado por afecciones médicas preexistentes, como las pérdidas proteicas, osteodistrofia renal, dislipidemia y ECV ${ }^{20}$. Incluso, la obesidad se relaciona con peor supervivencia del injerto, y la desnutrición con fracaso tardío del injerto ( $<5$ años), debido principalmente a la nefropatía de este ${ }^{5}$.

\section{Dislipidemia}

A los receptores de TR con dislipidemia es fundamental recomendar el consumo de una alimentación distribuida adecuadamente en grasas. A su vez, debe fomentarse la inclusión del -glucano de fibra soluble (avena, centeno, cebada, quinoa, amaranto, arroz integral, levaduras, trigo y maíz), ya que ha demostrado reducir el colesterol vinculado a lipoproteínas de baja densidad en las poblaciones que no reciben un trasplante. En el caso de las grasas polinsaturadas n-3, deben incluirse como fuentes vegetales y marinas ${ }^{28}$ (Tabla 2).

\section{Hipertensión}

La hipertensión es altamente prevalente, se estima que es de 4 a 5 veces mayor entre los receptores de TR en comparación a la población general, y es un 
factor de riesgo de insuficiencia del injerto, así como de ECV 29 .

Los pacientes con ERC en estadio 5 frecuentemente presentan hipertensión arterial sistémica como parte de las manifestaciones de la enfermedad. La relación con el TR en algunos casos condiciona la desaparición o una reducción de la gravedad de dicha entidad. Sin embargo, un elevado porcentaje de los receptores de TR persisten con hipertensión arterial posterior al trasplante, siendo un importante factor de riesgo para la disminución de la supervivencia del injerto ${ }^{30}$.

La etiología de la hipertensión arterial postrasplante es multifactorial y las causas más frecuentes son: rechazo crónico del injerto, administración crónica de inhibidores de calcineurina, presencia de riñones nativos, así como estenosis de la arteria del injerto renal ${ }^{30}$.

\section{Hipofosfatemia}

Las implicaciones clínicas de la hipofosfatemia postrasplante en términos del injerto y supervivencia del paciente no están claras. Por un lado, la hipofosfatemia puede reflejar una buena función del injerto asociada con una supervivencia prolongada de este y resultados cardiovasculares favorables ${ }^{31}$. Por otra parte, existe la posible exposición a largo plazo de altas concentraciones circulantes de hormonas fosfatúricas, factor de crecimiento de fibroblastos 23 y de hormona paratiroidea, las cuales podrían contribuir a un mayor riesgo de falla del injerto, así como de complicaciones cardiovasculares ${ }^{32}$.

\section{Hipomagnesemia}

La hipomagnesemia previa al trasplante puede considerarse como un factor de riesgo para desarrollar trastornos glucémicos posteriores. Los pacientes con niveles más bajos de magnesio $(\mathrm{Mg})$ en suero pretrasplante podrían tener un mayor riesgo de desarrollar glucosa alterada en ayuno o diabetes mellitus postrasplante. La incidencia varía del 2 al 50\% en TR, según los criterios utilizados para el diagnóstico, la duración del seguimiento y el régimen inmunosupresor. Este trastorno metabólico no solo aumenta el riesgo de ECV e infecciones, sino que también afecta la supervivencia del paciente y del injerto ${ }^{33}$.

Así mismo, se puede presentar con frecuencia posterior al trasplante, debido en parte a los regímenes inmunosupresores, incluidos los inhibidores de la calcineurina, que inducen pérdidas urinarias de $\mathrm{Mg}$. Aunque no se ha comprendido en su totalidad, se ha demostrado que los inhibidores de la calcineurina inducen una baja regulación de la expresión renal del factor de crecimiento epidérmico y TRMP6 (miembro 6 de la subfamilia de canales catiónicos receptores potenciales transitorios) en el túbulo colector distal, lo que conduce a una disminución en la reabsorción de $\mathrm{Mg}^{34}$.

Muchos otros factores influyen en la aparición de hipomagnesemia, como la expansión del volumen posterior al trasplante, la acidosis metabólica, RI, disminución de la absorción gastrointestinal en presencia de diarrea, baja ingesta de $\mathrm{Mg}$ y medicamentos como diuréticos o inhibidores de la bomba de protones ${ }^{34}$.

\section{Osteoporosis}

La osteoporosis y la osteopenia son una grave complicación que afecta entre el 50 y el $80 \%$ de los receptores; como consecuencia de ello, entre un 11 y un $40 \%$ sufren fracturas patológicas. Aunque se ha demostrado que la pérdida ósea es más elevada dentro de los primeros 6 a 18 meses postrasplante, Castillo et al. demostraron que una pérdida continua de masa ósea aumenta el riesgo de fracturas, hecho que empeora con la edad avanzada, deficiencia de calcio y vitamina $D$ en la dieta, inactividad física, dislipidemia y elevado peso corporal ${ }^{35}$.

En un análisis realizado por Cianciolo et al., la vitamina $D$ activa redujo los niveles de hormona paratiroidea y mejoró la presencia de trastorno mineral y óseo postrasplante, convirtiéndose en una terapia preventiva bien aceptada contra la pérdida ósea en los receptores de TR con osteopenia u osteoporosis ${ }^{36}$. Por otro lado, un estudio más reciente mostró que la vitamina $D$ y sus análogos pueden reducir la proteinuria en pacientes con $\mathrm{ERC}^{37}$.

Cabe señalar que sin vitamina $\mathrm{D}$ solo se absorbe del 10 al 15\% del calcio proveniente de la alimentación y aproximadamente el $60 \%$ del fósforo. La interacción de la vitamina 1,25-dihidroxivitamina $D$ con el receptor de vitamina $D$ aumenta la eficiencia de absorción intestinal de calcio del 30 al $40 \%$ y la absorción de fósforo aproximadamente en el $80 \%{ }^{38}$. Por lo tanto, se recomienda la administración de suplementos de calcio de 1,000 a 1,500 mg/día con 400 a 800 Ul/día de vitamina $D$, en conjunto con la abstinencia de consumo de tabaco y exceso de alcohol para ayudar a limitar la progresión o aparición de osteoporosis ${ }^{23}$.

\section{Actividad física}

Tanto la dieta como la actividad física son objetivos importantes en la intervención para la mejora en el 
estilo de vida de los pacientes posterior al trasplante. Es importante destacar que también se demostró que un programa de ejercicio supervisado conduce a mejoras similares en la fuerza muscular y la capacidad aeróbica en receptores de TR en comparación con individuos sanos ${ }^{39}$.

No obstante, para que la actividad física y el ejercicio resulten en un cambio favorable de un estado catabólico a un estado anabólico, es necesario considerar adecuadamente el estado nutricional, en particular en la ingesta proteica, y el estado metabólico, previniendo el aumento de peso después del trasplante y de esta manera, mejorando la calidad de vida de los receptores ${ }^{39}$.

\section{Conclusiones}

En la actualidad, el TR es una opción de tratamiento viable y con gran éxito para pacientes que viven con ERC terminal, no obstante, la evaluación perioperatoria del estado nutricional es un tema poco abordado por el personal de salud en general. Las guías actuales (KDIGO y KDOQI) mencionan la importancia de dicha intervención previa al TR.

El uso del MIS ha demostrado ser un tamizaje efectivo en cuanto a la identificación de pacientes con riesgo nutricional y a su vez es útil como predictor de mortalidad en estos pacientes. En diversos estudios se ha mostrado la importancia de una correcta intervención nutricional con una adecuada suplementación profiláctica para disminuir el riesgo de eventos desfavorables posteriores al trasplante, como el uso de suplementos de calcio de 1,000 a 1,500 mg/día con 400 a $800 \mathrm{UI} /$ día de vitamina $\mathrm{D}$, en conjunto con la abstinencia de consumo de tabaco y exceso de alcohol para limitar la progresión o aparición de osteoporosis. Sin embargo, se necesitan ensayos clínicos controlados sobre suplementación de micronutrimentos, así como estrategias nutricionales para mejorar el pronóstico en general de los pacientes sometidos a TR.

\section{Financiamiento}

El presente artículo no recibió fondos de instituciones públicas o privadas.

\section{Conflicto de intereses}

Los autores declaran no tener ningún conflicto de intereses.

\section{Responsabilidades éticas}

Protección de personas y animales. Los autores declaran que para esta investigación no se han realizado experimentos en seres humanos ni en animales.

Confidencialidad de los datos. Los autores declaran que en este artículo no aparecen datos de pacientes.

Derecho a la privacidad y consentimiento informado. Los autores declaran que en este artículo no aparecen datos de pacientes.

\section{Bibliografía}

1. Hori S, Ichikawa K, Morizawa $Y$, Gotoh D, Itami $Y$, Nakai $Y$, et al. Clinical significance of postoperative nutritional status as a prognostic factor in kidney transplant recipients. Transplant Proc. 2019;51(6):1763-72.

2. Fong JVN, Moore LW. Nutrition trends in kidney transplant recipients: The importance of dietary monitoring and need for evidence-based recommendations. Front Med. 2018;5(OCT):1-7.

3. Sabbatini M, Ferreri L, Pisani A, Capuano I, Morgillo M, Memoli A, et al. Nutritional management in renal transplant recipients: A transplant team opportunity to improve graft survival. Nutr Metab Cardiovasc Dis. 2019;29(4):319-24

4. Kluch M, Kurnatowska I, Matera K, Łokieć K, Puzio T, Czkwianianc E, et al. Nutrition trends in patients over the long term after kidney transplantation. Transplant Proc. 2020;52(8):2357-62.

5. Leal-Escobar G, Osuna-Padilla IA, Moguel-González B. Medical nutrition therapy in chronic kidney disease; from dialysis to transplant: A case report. Rev Esp Nutr Humana y Diet. 2016;20(2):80-7.

6. Sánchez MN, Merlo CA, Aguad ZR, Torino JR. Valoración e intervenciones nutricionales en pacientes en hemodiálisis. Rev Nefrol Dialisis Traspl. 2018;38(4):273-9.

7. Kalantar-Zadeh K, Kleiner M, Dunne E, Lee GH, Luft FC. A modified quantitative subjective global assessment of nutrition for dialysis patients. Nephrol Dial Transplant. 1999;14(7):1732-8.

8. Demirağ $A$, Kalayci M, Kantarci G, Gökçe Ö. Modified quantitative subjective global assessment of nutrition in patients on the renal transplant waiting list. Transplant Proc. 2009;41(1):108-11.

9. Kalantar-Zadeh K, Kopple JD, Block G, Humphreys MH. A malnutrition-inflammation score is correlated with morbidity and mortality in maintenance hemodialysis patients. Am J Kidney Dis. 2001;38(6):1251-63.

10. Carrero JJ, Thomas F, Nagy K, Arogundade F, Avesani CM, Chan M, et al. Global prevalence of protein-energy wasting in kidney disease: A meta-analysis of contemporary observational studies from the International Society of Renal Nutrition and Metabolism. J Ren Nutr. 2018;28(6):380-92

11. Ikizler TA, Burrowes JD, Byham-Gray LD, Campbell KL, Carrero JJ, Chan W, et al. KDOQI Clinical Practice Guideline for Nutrition in CKD: 2020 Update. Am J Kidney Dis. 2020;76(3):S1-S107.

12. Sukackiene D, Laucyte-Cibulskiene A, Vickiene A, Rimsevicius L, Miglinas $M$. Risk stratification for patients awaiting kidney transplantation: Role of bioimpedance derived edema index and nutrition status. Clin Nutr. 2020;39(9):2759-63.

13. Ryu H, Park HC, Kim H, Heo J, Kang E, Hwang YH, et al. Bioelectrical impedance analysis as a nutritional assessment tool in autosomal dominant polycystic kidney disease. PLoS One. 2019;14(4):1-18.

14. Kalista-Richards $M$. The kidney: Medical nutrition therapy-yesterday and today. Nutr Clin Pract. 2011;26(2):143-50.

15. Moreau K, Desseix A, Germain C, Barthe N, Bachelet T, Morel D, et al. Body composition in 98 patients awaiting kidney transplantation. Nutrition. 2014;30(2):186-91

16. Chan W, Bosch JA, Jones D, McTernan PG, Phillips AC, Borrows R Obesity in kidney transplantation. J Ren Nutr. 2014;24(1):1-12.

17. Tantisattamo E, Kalantar-Zadeh K, Halleck F, Duettmann W, Naik M, Budde K. Novel approaches to sarcopenic obesity and weight management before and after kidney transplantation. Curr Opin Nephrol Hypertens. 2021;30(1):14-26.

18. Martins C, Pecoits-Filho R, Riella MC. Nutrition for the post-renal transplant recipients. Transplant Proc. 2004;36(6):1650-4.

19. Dionigi $P$, Alessiani M. Nutrition of the kidney transplant recipients. En: Orlando G, Remuzzi G, Williams DF, editores. Kidney transplantation, bioengineering, and regeneration. Kidney transplantation in the regenerative medicine era. Elsevier; 2017. pp. 677-683.

20. Chitra U, Sunitha Premalatha K. Nutritional management of renal transplant patients. Indian J Transplant. 2013;7(3):88-93. 
21. Chadban S, Chan M, Fry K, Patwardhan A, Ryan C, Trevillian P, et al. Protein requirement in adult kidney transplant recipients. Nephrology. 2010;15(Suppl. 1):71-4.

22. Anbar R, Lipa R, Zinger $P$, Mor $E$. The role of nutritional support in transplant recipients: Two case reports. Transplant Proc. 2003;35(2):614-6.

23. Tritt L. Nutritional assessment and support of kidney transplant recipients. $\mathrm{J}$ Infus Nurs. 2004;27(1):45-51

24. Cano NJM, Aparicio M, Brunori G, Carrero JJ, Cianciaruso B, Fiaccadori E, et al. ESPEN Guidelines on Parenteral Nutrition: Adult Renal Failure. Clin Nutr. 2009;28(4):401-14.

25. Chan M, Patwardhan A, Ryan C, Trevillian P, Chadban S, Westgarth F, et al. Evidence-based guidelines for the nutritional management of adult kidney transplant recipients. J Ren Nutr. 2011;21(1):47-51.

26. Paul R, Hynes M, Lew SQ. Nutrition in kidney transplant recipients to prevent downstream health issues. J Kidney Care. 2019;4(5):252-60

27. Nicoletto BB, Souza GC, Gonçalves LF, Costa C, Perry IS, Manfro RC Leptin, insulin resistance, and metabolic changes 5 years after renal transplantation. J Ren Nutr. 2012;22(4):440-9.

28. Chadban S, Chan M, Fry K, Patwardhan A, Ryan C, Trevillian P, et al. Nutritional management of dyslipidaemia in adult kidney transplant recipients. Nephrology. 2010;15(Suppl. 1):62-7.

29. van den Berg E, Engberink MF, Brink EJ, van Baak MA, Gans ROB Navis $\mathrm{G}$, et al. Dietary protein, blood pressure and renal function in renal transplant recipients. Br J Nutr. 2013;109(8):1463-70.

30. Chew-Wong A, Alberú-Gómez J, Abasta-Jiménez M, Márquez-Díaz F, Correa-Rotter R. Hipertensión arterial postrasplante renal: factores de riesgo asociados e influencia en la supervivencia del injerto renal. Gac Med Mex. 2005;141(6):461-8.
31. Huber L, Naik M, Budde K. Frequency and long-term outcomes of post-transplant hypophosphatemia after kidney transplantation. Transpl Int. 2013;26(10):94-6.

32. van Londen M, Aarts BM, Deetman PE, van der Weijden J, Eisenga MF Navis G, et al. Post-transplant hypophosphatemia and the risk of death-censored graft failure and mortality after kidney transplantation. Clin $J$ Am Soc Nephrol. 2017;12(8):1301-10.

33. Alamdari A, Asadi G, Minoo FS, Khatami MR, Gatmiri SM, Dashti-Khavidaki $\mathrm{S}$, et al. Association between pre-transplant magnesemia and post-transplant dysglycemia in kidney transplant recipients. Int $\mathrm{J}$ Endocrinol Metab. 2020;18(1):1-7.

34. Garnier AS, Duveau A, Planchais M, Subra JF, Sayegh J, Augusto JF. Serum magnesium after kidney transplantation: A systematic review. Nutrients. 2018;10(6):1-12.

35. Castillo RF, Gallegos RF, Amaro MPP, de la Rosa RJE. Valoración del perfil lipídico y de la densidad mineral ósea en pacientes trasplantados renales. Nutr Hosp. 2015;31(6):2503-10.

36. Cianciolo G, Galassi A, Capelli I, Angelini ML, La Manna G, Cozzolino M. Vitamin $D$ in kidney transplant recipients: Mechanisms and therapy. Am J Nephrol. 2016;43(6):397-407.

37. de Borst MH, Hajhosseiny R, Tamez H, Wenger J, Thadhani R, Goldsmith DJA. Active vitamin D treatment for reduction of residual proteinuria: A systematic review. J Am Soc Nephrol. 2013;24(11):1863-71.

38. Bouillon R, Norman AW, Lips P. Vitamin D deficiency. N Engl J Med. 2007;357(19):1980-2.

39. Klaassen G, Zelle DM, Navis GJ, Dijkema D, Bemelman FJ, Bakker SJL, et al. Lifestyle intervention to improve quality of life and prevent weight gain after renal transplantation: Design of the Active Care after Transplantation (ACT) randomized controlled trial. BMC Nephrol. 2017;18(1):1-13. 